\title{
Unicidade: Proposta para uma cidadania ativa no espaço urbano aumentado do bairro de Casa Amarela
}

\author{
Unicidade: Proposal to an active citizenship in the \\ augmented urban space of Casa Amarela neighbourhood
}

Madyana Torres, Hans Waechter

design participativo, cidade aumentada, vizinhança, comunidade participatory

design, augmented

city, neighbourhood, community

\begin{abstract}
Áreas públicas urbanas refletem mudanças em estruturas socioeconômicas e políticas de uma população. Conceitos como os de Cidade Aumentada revelam como atualmente esse espaço é progressivamente transformado e medido, bem como o crescimento de sua importância. Dado esse contexto, este trabalho objetivou estudar como o sentimento de proteção e familiaridade podem ser estimulados e como o designer pode lidar com a complexidade deste cenário. Para tanto, um projeto foi proposto para o bairro de Casa Amarela, no Recife. Com foco em como estimular o engajamento do atual grupo de moradores ao coletivo Casa Amarela Saudável e Sustentável, foram utilizadas técnicas de Design Participativo para a investigação, ideação e a criação do projeto.
\end{abstract}

Urban public spaces reflect changes in socioeconomic and political structures of a population. Concepts such as Augmented City reveal how this space is currently being progressively transformed and measured, as well as the growth of its importance. Given this context, this study investigates how to stimulate the feeling of protection and familiarity and how the designer can handle the complexity of this scenario. To that end, a project was proposed for the Casa Amarela neighbourhood, in Recife. Focusing on how to stimulate the engagement of the current group of residents to the collective Casa Amarela Saudável e Sustentável, techniques of Participatory Design were used for research, ideation and project development.

\section{Introdução}

A relação de habitantes entre si e com a cidade é um conjunto de informações em constante mudança, observada através de ambientes públicos. Atuar nessa relação pode trazer consequências positivas, como a conscientização de problemas numa comunidade e decisões que considerem os fatores humanos de maneira mais coerente com o contexto em que estão inseridos. 
Através de um trabalho de conclusão de curso, foi proposto um projeto para entender como moradores engajam-se, coletar ideias para estimular este processo e observar uma reação ao conceito de Cidade Aumentada através dos métodos de design participativo, demonstrando como esta abordagem pode contribuir para a atuação do designer em temas complexos.

\section{Interações no Espaço Urbano}

Uma cidade pode ser entendida como um conjunto de sistemas complexos, com atores variados e com uma enormidade de interações entre eles (Smyth et al., 2013). A evolução das cidades é hoje discutida através da interferência tecnológica nas relações entre esses atores e o sistema que os conecta, manifesta através de conceitos como Smart Cities e Augmented Cities - respectivamente Cidades Inteligentes e Cidades Aumentadas.

\subsection{Aumentando a cidade}

O termo Smart Cities é usado para descrever cidades onde o uso da tecnologia torna a infrae strutura e os serviços públicos mais eficientes. O conceito, embora recente, já é alvo de críticas direcionadas à corporativização dos projetos de desenvolvimento, consequências de uma abordagem focada nas instituições e ignorando o papel ativo do indivíduo (De Lange; De Waal, 2013).

Os autores indicam a Cidade Aumentada como alternativa. Na noção da cidade como propriedade, o papel da tecnologia muda de gerenciamento para construção. Os recursos tecnológicos podem ser usados para criar um relacionamento com o ambiente e com outros habitantes, incentivando o sentimento de coletividade. O cidadão transforma-se em cocriador, corresponsável pelo que acontece ao seu redor e adicionando sua perspectiva ao contexto. E o espaço público é potencialmente onde cidadãos com diferentes perspectivas podem interagir.

\section{Heterotopias e o Espaço Público}

1 Potencialmente um espaço terciário, que ancora a vida comunitária. Cf. OLDENBURG, RAY. 1989. The Great Good Place.
O espaço público urbano é definido pelo Urbanismo como uma área dentro da cidade para uso coletivo que pertence, ao mesmo tempo, às autoridades e sociedade (Junior, 2014). Para entender as dimensões do espaço público, Henri Lefebvre (1991) divide o conceito em:

- Espaço prático: oriundo do uso cotidiano e experimentado pelo cidadão;

- Espaço representativo: quando é descrito para terceiros que não desfrutam do local 
- Espaço representacional: quando criado no imaginário através de ícones e símbolos, no aspecto emocional.

Adiante em seu estudo, Lefebvre aponta que existem contradições entre os elementos abstratos e concretos de um espaço público. Heterotopia, conceito criado por Foucault (1986), é usado para descrever espaços contraditórios como estes, que possuem diversas camadas de significado e relacionamentos nem sempre claros para todos que se referem a ele. A noção de Propriedade torna-se então um meio de legitimar o direito à cidade, capaz de promover alianças entre cidadãos para comunicação dessas diferenças de significados.

\section{0 contexto: Casa Amarela}

A vizinhança escolhida para este estudo localiza-se no bairro de Casa Amarela, região norte do Recife. O bairro, um dos maiores e mais antigos, foi escolhido pelas diversas camadas de importância econômica, política, histórica e afetiva.

Hoje, a disparidade entre o desenvolvimento de partes diferentes do bairro chama à atenção de estudiosos e moradores. Segundo o estudo do IBGE em 2011, o centro de Casa Amarela possui IDH similar aos de países como a Nova Zelândia, enquanto regiões de morro assemelham-se à regiões como o Tadjiquistão (Markman, 2011).

Figura 1 Morro da Conceição (Fonte: Adauto Júnior)

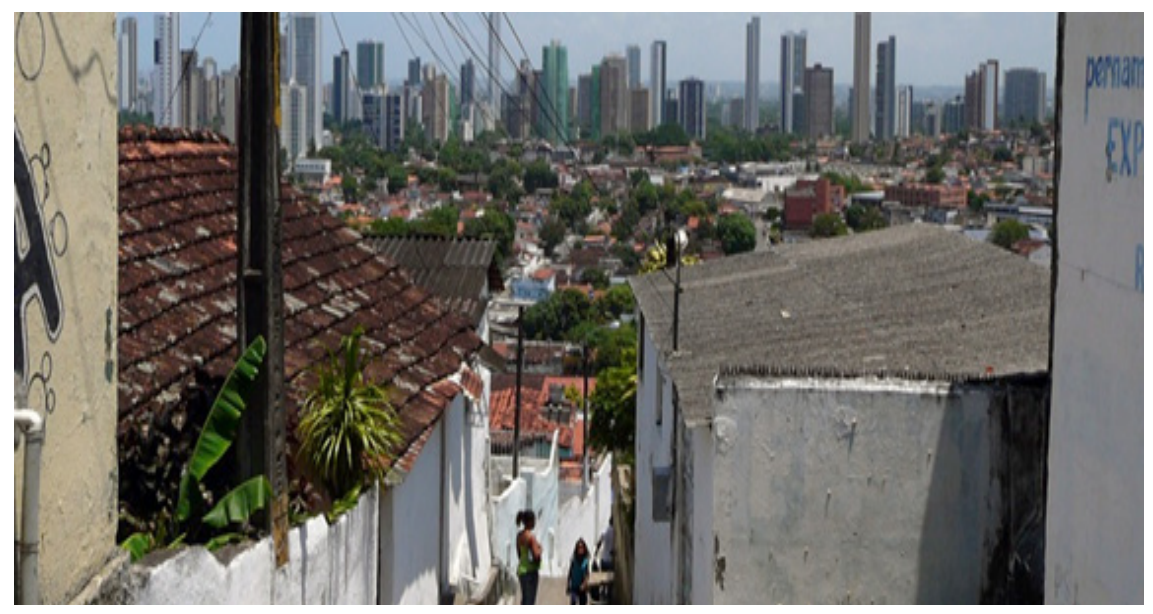

\section{Coletivo Casa Amarela Saudável e Sustentável}

Diversas iniciativas populares surgiram em reação ao crescimento desordenado da região. Casa Amarela Saudável e Sustentável é uma delas: com pequenas sugestões acatadas pela Prefeitura, o coletivo organizado através de redes sociais ganhou atenção na luta para estabelecimento de um Plano Diretor para o bairro. Por essa razão o coletivo foi escolhido como um dos colaboradores deste trabalho. 
Figura 2 Coletivo Casa Amarela Saudável e Sustentável (Fonte: Souza, 2015).

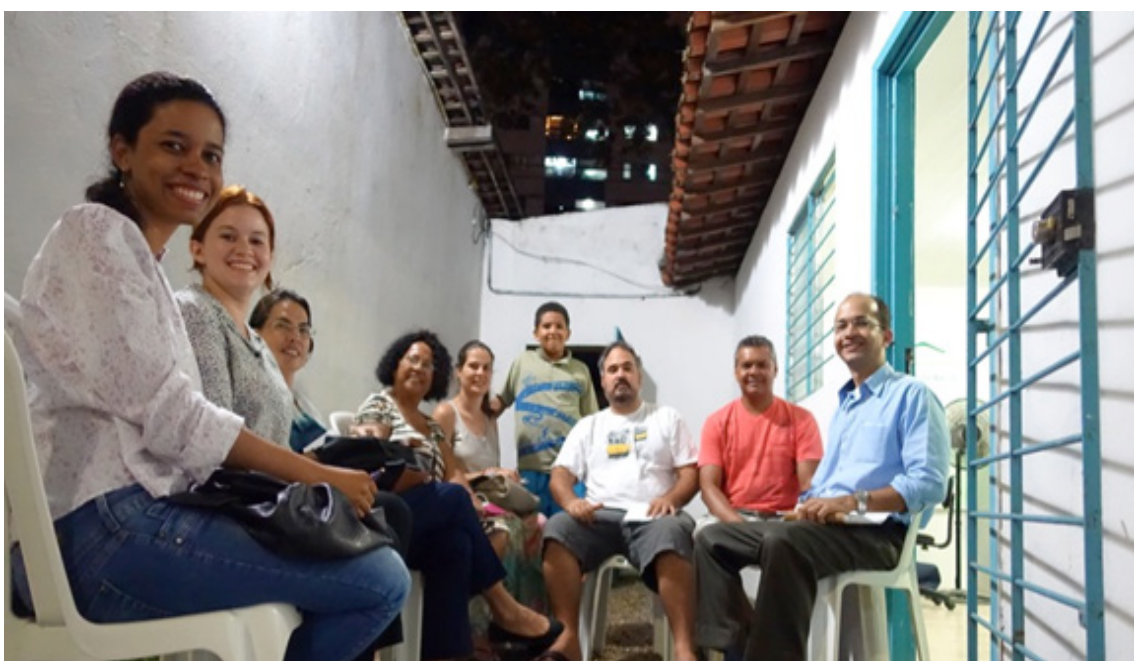

Design Participativo: estimulando a análise e reflexão numa comunidade

O Design Participativo foi escolhido pelo seu caráter inclusivo. Spinuzzi (2005) o define como um conjunto de métodos que inclui as pessoas relacionadas ao projeto em sua concepção. Enquanto outras metodologias utilizam uma pesquisa proveniente de outros campos para iniciar a fase de projeto, o Design Participativo inclui os usuários durante todo o projeto. Pesquisa e ideação estão interligadas e a participação dos usuários não possui somente o papel de confirmação, visto que é essencial à geração da ideia.

\section{Investigando o contexto}

Para analisar a relação do público geral e informar-se sobre o bairro, foram conduzidas entrevistas, divididas em três fases:

- Triagem: identificando os participantes que de fato moravam ou visitavam o bairro com frequência;

- Funções práticas: identificando as relações desses participantes com locais do bairro, uso das áreas e compartilhamento de problemas:

- Sentimento e história: entrevistas mais longas e abertas com um representante do grupo de moradores e outro grupo de visitantes.

Todas as entrevistas foram realizadas online, com exceção das relacionadas aos aspectos emocionais. A pesquisa foi realizada com 97 participantes no total, selecionados de acordo com o tempo que permaneciam no bairro. As atividades do coletivo também foram acompanhadas em reuniões com participação variável entre de 6 a 23 pessoas. 


\section{Ideação: Criando o Boundary Object}

Para criar um suporte à interações entre membros, a ideação ambicionou criar um objeto fronteiriço ou Boundary object, conceito introduzido por Susan Star (1989) que descreve um elemento comum onde práticas de diferentes mundos sociais encontram-se e são coordenadas.

Os seguintes métodos de design participativo foram usados ("FROG - Collective Action"):

- Mapa mental de Impacto: criado com os participantes e usado para definir os valores e alcance das ações do coletivo. Através desse mapa foram classificadas as relações de acordo com as estruturas sugeridas por Weber (apud Blokland, 2003).

- Problem Cards: Reunindo informações do exercício anterior, os participantes sintetizaram em uma sentença problemas relacionados ao bairro e a possível razão desse problema permanecer.

- Brainwriting 6-3-5: O exercício anterior apontou a falta de comunicação e mobilização como fatores que impediam a solução de alguns problemas. Esse resultado foi tema de uma sessão de geração de ideias através do método 6-3-5. Nesse método, tradicionalmente seis participantes escrevem ou desenham três ideias num papel durante cinco minutos e passam adiante ao próximo membro, recebendo as ideias de outro.

Figura 3 Mapa de Relações (Fonte: Souza, 2015 com método de Weber apud Blokland, 2003).

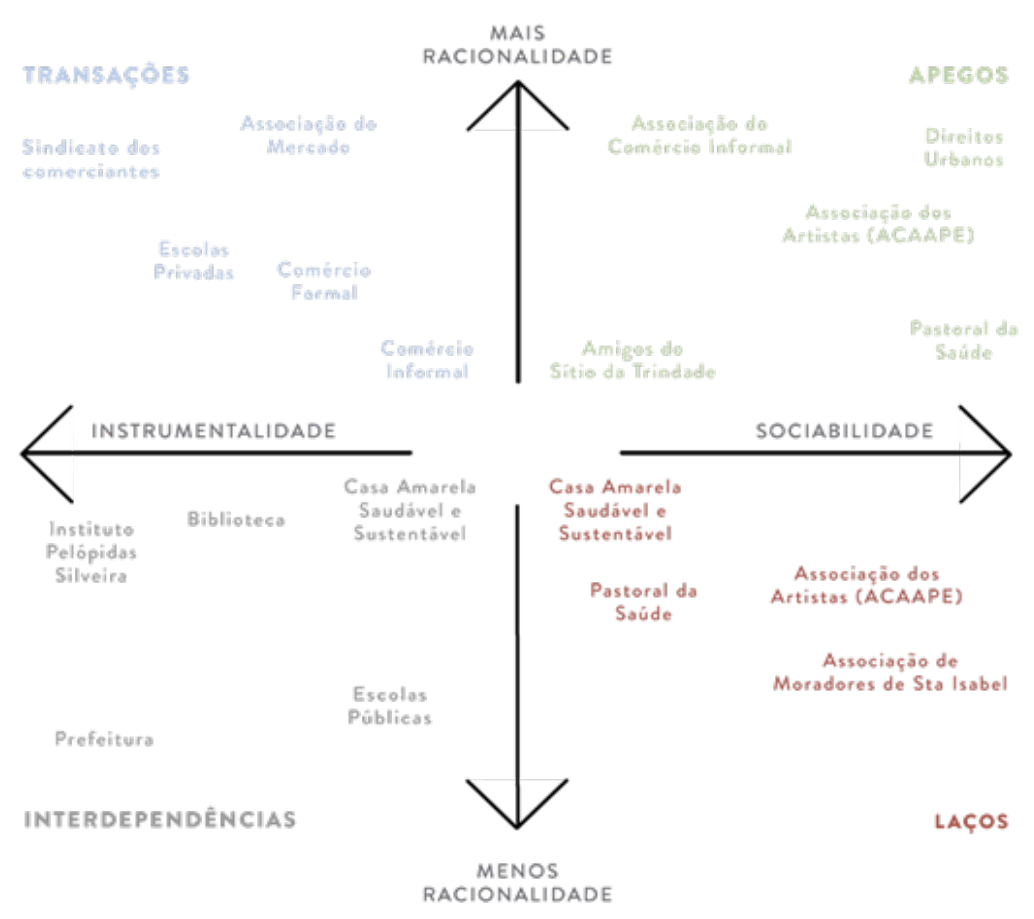




\section{Resultados preliminares}

Durante o processo o grupo apontou como prioridades a democratização da tecnologia, o engajamento de moradores e a dificuldade de manter plataformas colaborativas fiéis ao seu propósito. O projeto a seguir foi refinado levando em consideração o aspecto mais relevante e com maior adesão do grupo.

\section{Unicidade: diálogo e concordância entre moradores}

Unicidade é uma plataforma criada para garantir a expressão de moradores de um bairro, divulgar ações de iniciativas populares e conectar-se com moradores não-engajados. O nome do projeto refere-se à unificação do ambiente urbano e ao processo de concordância entre os integrantes de uma comunidade que culminaria num estado harmônico. Unicidade permite que a comunidade escreva e escolha as mensagens mais relevantes, que serão projetadas em um espaço público e num website. Membros dos coletivos e iniciativas populares estarão acessíveis para contribuir, orientar e atualizar os moradores com as atividades mais recentes.

Figura 4 Funcionamento do projeto (Fonte: Souza, 2015).

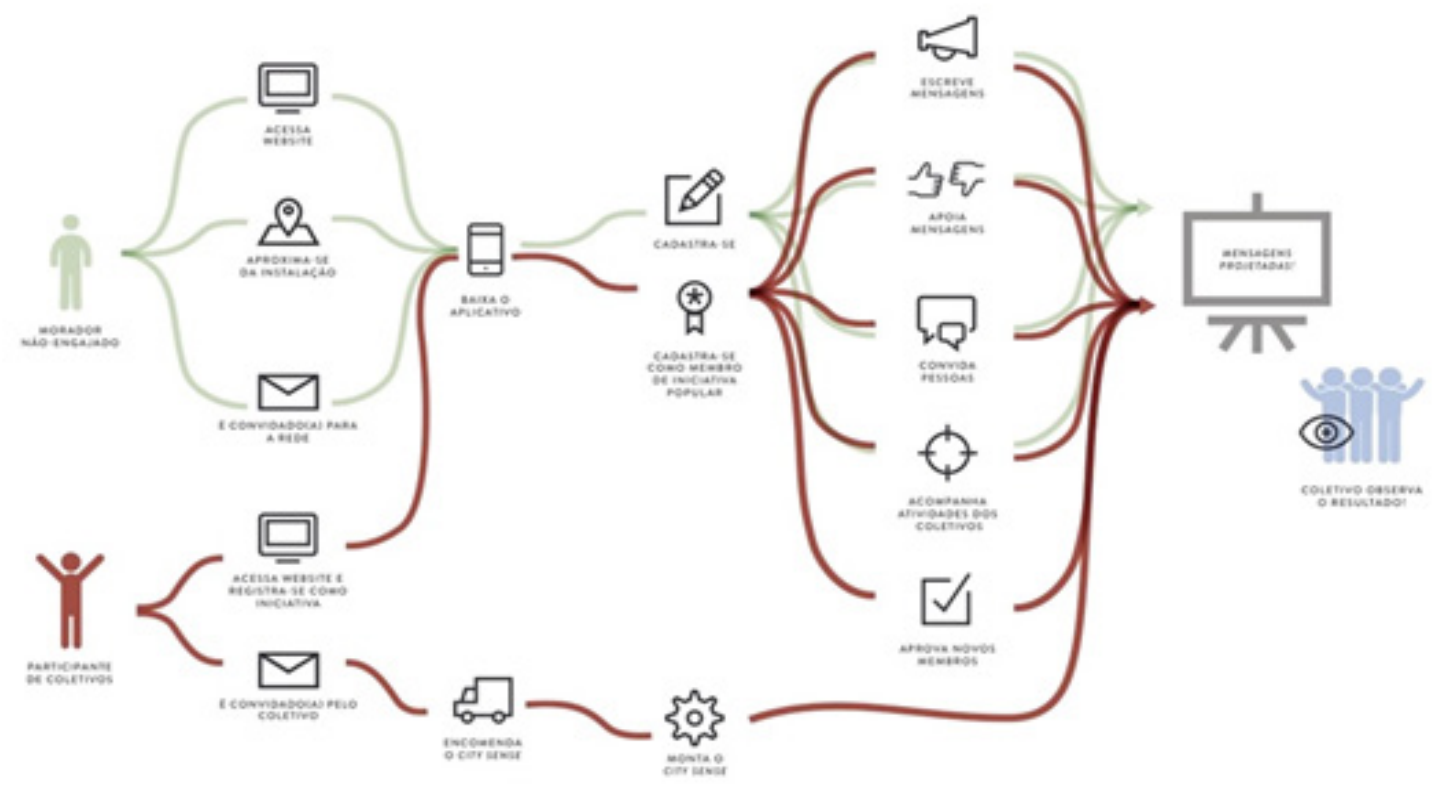

\section{Instalação Urbana}

Através de um projetor, mensagens escritas e escolhidas através de um aplicativo podem ser visualizadas por todos ao redor. Desse modo o espaço público escolhido é transformado num suporte para as mensagens dos membros da comunidade. 
O Mercado Público de Casa Amarela foi apontado pelas pesquisas como um dos locais favoritos dos moradores e por isso escolhido para demonstração. Como o Mercado é bastante movimentado, a interação através de um aplicativo foi escolhida como principal para não atrapalhar as tarefas de quem passa no local.

Figura 5 Projeção no Mercado Público. (Fonte: por Adauto Júnior e edição por Souza, 2015).

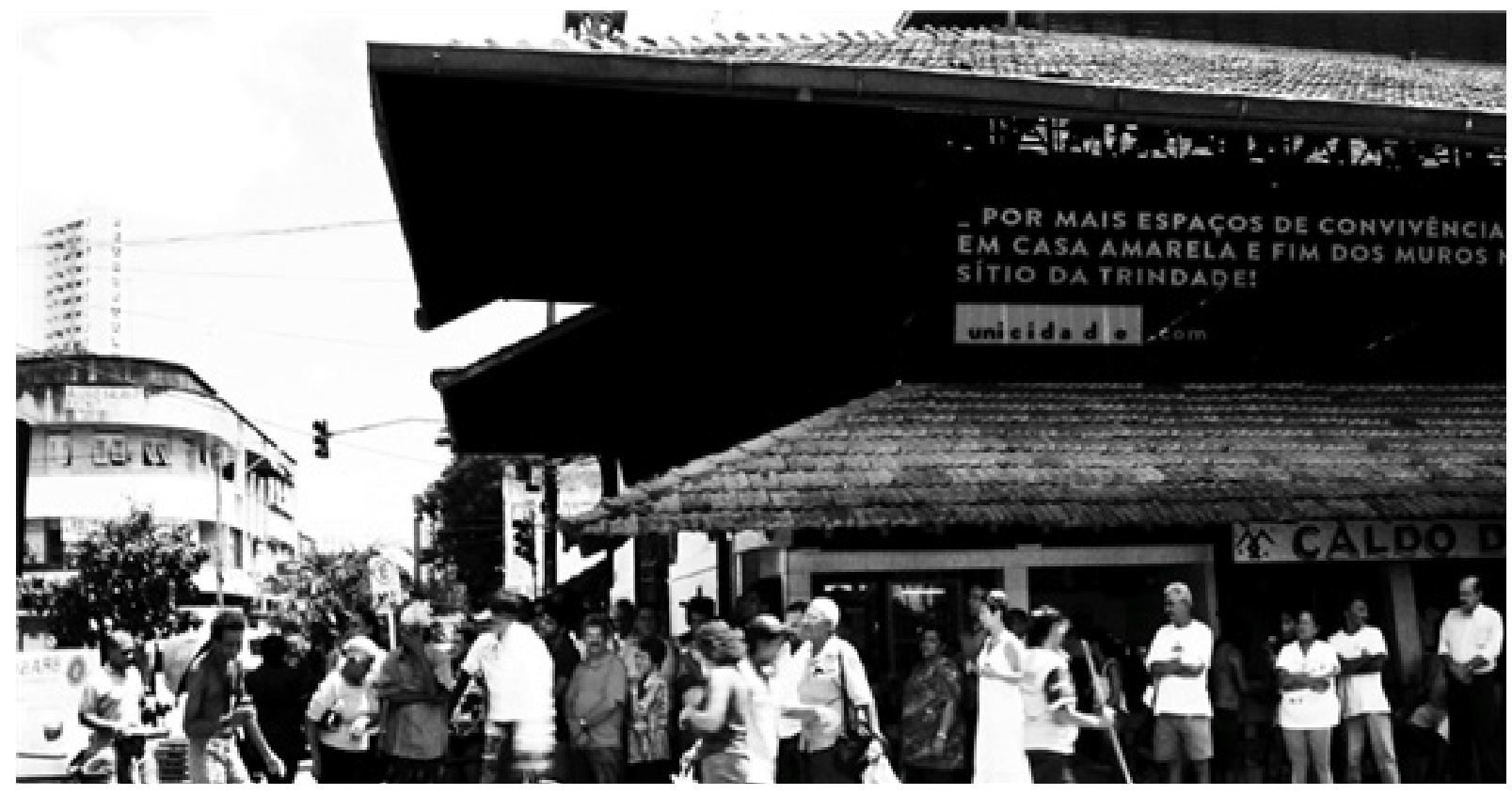

Aspectos Técnicos

O artefato é composto por projetor, suporte a rede $4 \mathrm{G}$, GPS e um processador. Atualmente, pequenos projetores com alta resolução já estão disponíveis, bem como computadores de hardware aberto, como Raspberry Pi.

Para tornar possível a instalação em diversos cenários, seriam oferecidas combinações de equipamentos. O processo de aquisição e montagem do artefato é inspirada na Fabricação Digital e movimento Maker, um importante marco para a independência produtiva e compartilhamento de ideias (Pellegrini, 2014).

\section{Meios de Interação}

Os membros do coletivo aprovariam os membros subsequentes com o aplicativo. Cabe a eles inserir mensagens relacionadas aos interesses da comunidade e disponibilizarem-se para interações presenciais, através da transmissão voluntária de sua localização.

A instalação pode receber mensagens de moradores e frequentadores de Casa Amarela. Os moradores podem enviar mensagens anonimamente, conectados ao aplicativo. Frequentadores podem utilizar o website e deixar suas mensagens somente quando ao redor da instalação. 
2 Vídeo pode ser acessado através da URL [https:// vimeo.com/126075539].
Figura 6 Aplicativo e Website (Fonte: Souza, 2015).

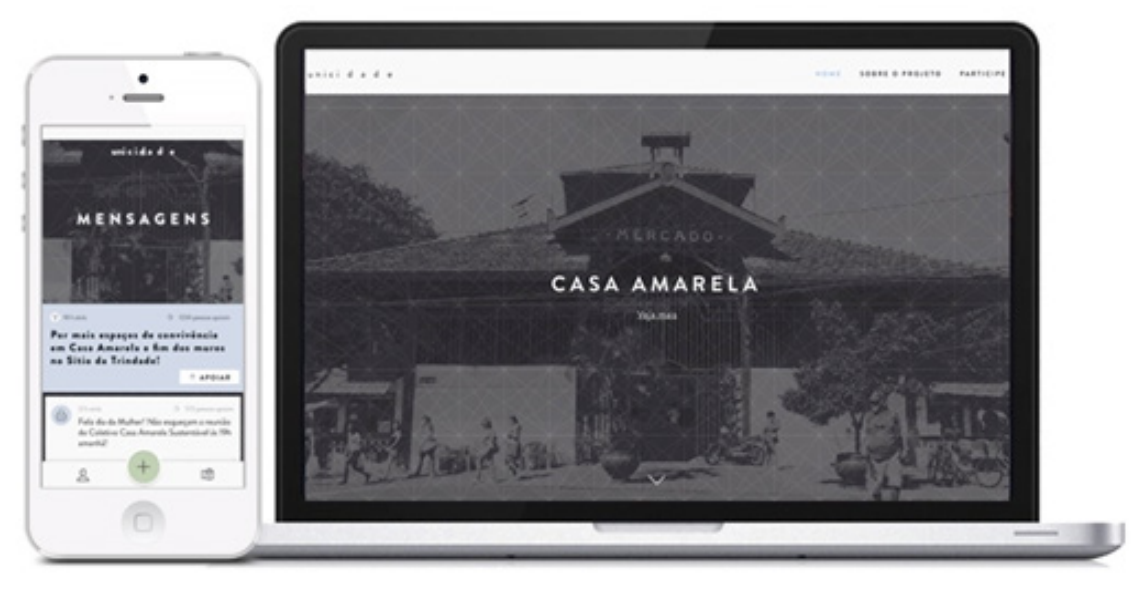

O projeto foi apresentado para o coletivo e consolidado em um vídeo².

\section{Considerações Finais}

Comunidades existem sob regras políticas e cívicas, mas sobretudo os sentimentos de familiaridade, segurança e pertencimento que a definem. Durante este trabalho, foi possível observar a iniciativa de um grupo de moradores que concorda com essa afirmação e tenta hoje transformar sua vizinhança.

O papel do designer dentro de contextos como esse é especialmente fortalecido com o Design Participativo, por este facilitar a tomada de decisões, mesclar pesquisa e ideação e fornecer uma visão mais profunda de um problema, nas palavras e experiências de quem passa por ele. Serve também aos participantes, por empoderar e ampliar sua atuação, garantindo que o resultado será apresentado com uma perspectiva mais familiar e cuidadosa. Neste processo em específico, o grupo participante guiou o uso da tecnologia, o que demonstra um nível de familiaridade das comunidades e organizações atuais - caminhando em direção às cidades aumentadas através das mobilizações através de redes sociais. A inclusão da tecnologia como educação básica pode estimular ainda mais a participação popular nesse espaço público virtual.

Espera-se que esse trabalho tenha esclarecido que apresentar soluções para um cenário tão complexo é um desafio, que pode ser simplificado quando escuta-se quem vive no contexto pesquisado. Os limites do papel e função do designer acabam por serem transformados: de solucionador para mediador de questões mais amplas, tornando o design numa linguagem comum para diferentes interesses emergirem com soluções. 


\section{Referências}

BLOKLAND, T. 2003. Urban Bonds: Social Relationshipts in an Inner City Neighbourhood. Cambridge: Polity.

DE LANGE, M.; DE WAAL, M. 2013. Owning the city: New media and citizen engagement in urban design. First Monday, v. 18, n. 11.

FOUCAULT, M. 1986. Of Other Spaces. Diacritics, v.16, n.1: 22-27.

FROG - Collective Action. In: < http://www.frogdesign.com/work/frogcollective-action-toolkit.html>, 04/11/2014.

JUNIOR, O.S. 2014. Urban common space, heterotopia and the right to the city: Reflections on the ideas of Henri Lefebvre and David Harvey. urbe: Revista Brasileira de Gestão Urbana, v.6, n.2: 146-157.

LEFEBVRE, H. 1991. The Production of Space: 454. Oxford: Blackwell.

MARKMAN, L. No Recife, moradores de Casa Amarela sofrem com falta de esgoto. In: <http://g1.globo.com/pernambuco/noticia/2011/12/no-recifemoradores-de-casa-amarela-sofremcom-falta-de-esgoto.html>, 08/12/2014.

PELLEGRINI, B. Fabricação digital concretiza ideias de arquitetos e designers. In: <http://www5.usp.br/4837/tecnologia-de-fabricacao-digital-concretizaideias-de-arquitetos-edesigners/>. 30/11/2014.

SMYTH, M., HELGASON, I., ET AL. 2013. UrbanIxD : Designing Human Interactions in the Networked City. In: $\mathrm{CHI}$ '13 Extended Abstracts on Human Factors in Computing Systems on - CHI EA '13. New York, USA: ACM Press: 2533 .

SOUZA, M. 2015. Unicidade: Proposta para uma cidadania ativa no espaço urbano aumentado do bairro de Casa Amarela. Trabalho de conclusão de curso (graduação). Departamento de Design, Universidade Federal de Pernambuco, Recife.

SPINUZZI, C. 2005. The Methodology of Participatory Design. In: Technical Communication, v.52, n.2: 163-174.

\section{Sobre os autores}

\section{Madyana Torres de Souza}

Bacharel em Design, UFPE, Brasil <madytorres@gmail.com>

\section{Hans da Nóbrega Waechter}

PhD, UFPE, Brasil <hnwaechter@terra.com.br>

Artigo recebido em 09 set. 2015,

aprovado em 21 set. 2015. 